\title{
Large-scale Optimization of Contoured Beam Reflectors and Reflectarrays
}

Borries, Oscar; Sørensen, Stig B.; Jørgensen, Erik; Zhou, Min; Andersen, Martin Skovgaard; Sokoler, Leo Emil

\section{Published in:}

Proceedings of the 2016 IEEE Antennas and Propagation Society International Symposium (APSURSI)

Link to article, DOI:

10.1109/APS.2016.7696515

Publication date:

2016

Document Version

Peer reviewed version

Link back to DTU Orbit

Citation (APA):

Borries, O., Sørensen, S. B., Jørgensen, E., Zhou, M., Andersen, M. S., \& Sokoler, L. E. (2016). Large-scale Optimization of Contoured Beam Reflectors and Reflectarrays. In Proceedings of the 2016 IEEE Antennas and Propagation Society International Symposium (APSURSI) (pp. 1617-1618). IEEE.

https://doi.org/10.1109/APS.2016.7696515

\section{General rights}

Copyright and moral rights for the publications made accessible in the public portal are retained by the authors and/or other copyright owners and it is a condition of accessing publications that users recognise and abide by the legal requirements associated with these rights.

- Users may download and print one copy of any publication from the public portal for the purpose of private study or research.

- You may not further distribute the material or use it for any profit-making activity or commercial gain

- You may freely distribute the URL identifying the publication in the public portal 


\section{Large-scale Optimization of Contoured Beam Reflectors and Reflectarrays}

\author{
Oscar Borries, Stig B. Sørensen, \\ Erik Jørgensen, Min Zhou \\ TICRA, Copenhagen, Denmark \\ $\{$ ob,sbs,ej,mz $\} @$ ticra.com
}

\author{
Martin S. Andersen, Leo Emil Sokoler \\ Technical University of Denmark \\ Kgs. Lyngby, Denmark \\ \{mskan,leso\}@dtu.dk
}

\begin{abstract}
Designing a contoured beam reflector or performing a direct optimization of a reflectarray requires a mathematical optimization procedure to determine the optimum design of the antenna. A popular approach, used in the market-leading TICRA software POS, can result in computation times on the order of days, due to the optimization algorithm. The present paper discusses recent improvements, allowing reductions in optimization time by two orders of magnitude or more on several application examples.
\end{abstract}

\section{INTRODUCTION}

The design of reflectors and reflectarrays for geostationary contoured beam applications can require an optimization of the reflector surface or reflectarray elements to achieve the desired coverage. The mathematical algorithms in the optimization process constitute, for electrically large scatterers, a major time requirement in the design process, often being much more costly than the electromagnetic computations.

The optimization problem to be solved can be expressed as

$$
\begin{array}{cl}
\min _{\bar{X}} . & F(\bar{X})=\max \left(F_{1}(\bar{X}), F_{2}(\bar{X}), \ldots, F_{m}(\bar{X})\right), \\
\text { s.t. } & \bar{A} \bar{X} \leq \bar{B}
\end{array}
$$

where the $F$ function represents the largest discrepancy over the $m$ residual functions $F_{i}$ in the coverage. $F_{i}$ is the difference between the attained value and the desired goal at the $i$ 'th station in the coverage. $\bar{X}$ are the $n$ variables of interest, determining the shape of the reflector surface or the parameters of each of the reflectarray elements. The constraints (1b), where $\bar{B}$ contains $p$ elements, can specify constraints such that the solution is physically realizable, e.g., avoiding rapid surface variations or ensuring that reflectarray elements do not overlap.

\section{Algorithm}

The general framework of the algorithm is described in detail in the paper by Hald [1], and is illustrated in Algorithm 1. The main computational bottleneck of Algorithm 1 is the process involved in finding the direction $\Delta \bar{X}$ and the electromagnetic computation required to find $T$. For cases where $m, n$ and/or $p$ is large, finding $\Delta \bar{X}$ requires at least an order of magnitude more computation time than finding $T$.

The main problem with the existing algorithm is that finding $\Delta \bar{X}$ requires maintaining a factorization of a constantly

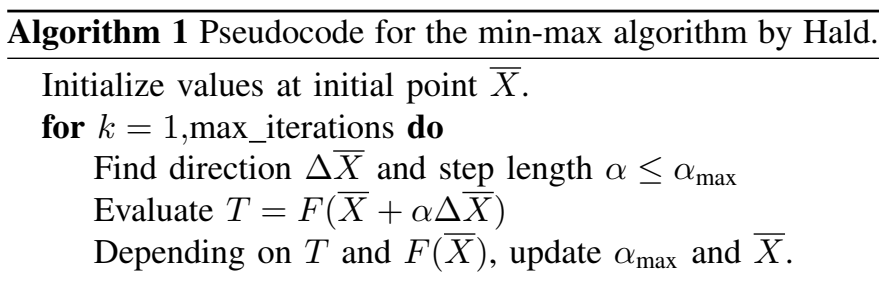

expanding matrix, adding a single row and column for each step. This is a slow but very accurate approach that it is inherently single-threaded, thus leaving no options for adapting the algorithm for modern computer architectures. Finally, it requires a large number of matrix-vector products, which can be very time-consuming for large cases.

The new optimization approach avoids this by using an interior-point solver to find $\Delta \bar{X}$ using large steps along the central path of the linearized problem. By taking large steps that activate multiple functions and constraints each time, we achieve a faster, easily parallelized implementation, which can be made sufficiently accurate by adjusting the tolerances of the interior-point solver. In practice, the difference in the converged value of $F$ between the new optimization approach and that of Hald is typically less than $0.01 \mathrm{~dB}$.

\section{RESULTS}

To illustrate the performance of the new algorithm, we consider two applications, namely the design of a shaped reflector and a reflectarray. All computations are performed on a Dual Intel Xeon E5-2690 2.9 GHz computing node.

\section{A. Shaped Reflector}

We consider a large shaped offset-fed reflector with coverage of the Continental United States plus Hawaii. The reflector has a circular rim with a diameter of $1 \mathrm{~m}$ and operates at $f=60 \mathrm{GHz}$, implying that the electrical size is $D=200 \lambda$. The surface of the reflector is defined as a paraboloid plus a surface defined as a set of splines - the unknowns for the problem are the spline coefficients. For this reflector, we choose $n=5225$ spline variables, and the coverage region is covered with $m=6642$ stations. We have no constraints, and perform 500 iterations, ignoring the fact that the optimization has not yet converged at the end of those 500 iterations. 
TABLE I

RESULTS FROM THE OLD AND NEW ALGORITHM WHEN APPLIED TO THE SHAPED REFLECTOR FROM SECTION III-A

\begin{tabular}{l|cc} 
& Optimization time & Optimum $F$ \\
\hline Old algorithm & 18:12 Hours & -2.09 \\
New algorithm & 5:46 Minutes & -2.11 \\
\hline
\end{tabular}

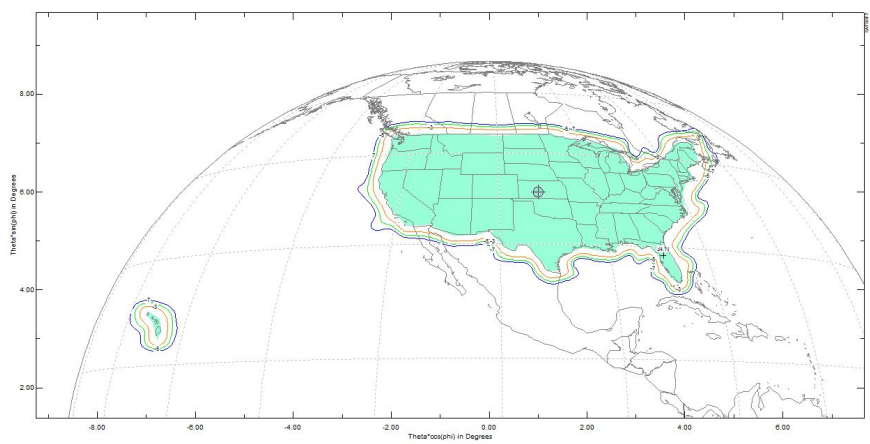

Fig. 1. Coverage of the reflector used in Section III-A along with the optimized pattern.

As shown in Table 1 the reduction in time spent by the optimization algorithm is a factor of 190 , achieving effectively the same result. Both algorithms evaluate the objective function $F$ once per iteration, and the time spent evaluating the objective function 500 times is 5:51 minutes, such that the total time spent solving the problem is $18: 18$ hours for the old algorithm and 11:37 minutes for the new. The coverage as well as the pattern from the optimized reflector is shown in Fig. 1

\section{B. Shaped Reflector with Constraints}

Constraints are often imposed on the final design. Such constraints can be on the variations in the surface curvature of the reflector, due to limits imposed by the manufacturing process, as well as on the size of the reflector in cases where the installed size has to be within certain limits.

A common approach when working towards a realistic antenna design is to perform the optimization without any constraints, as was done in the previous example, and then use that result as a starting guess for a new optimization procedure with constraints. The rule of thumb is that the curvature constraints should be sampled four times per spline. Further, linearizing the surface constraints increases the number of constraints by a factor of 8 , yielding a constraint matrix with roughly $128 n$ rows, depending on the reflector rim.

For our specific case, imposing the constraint that the local radius of curvature of the reflector has to be at least $0.1 \mathrm{~m}$ yields $p=639200$ constraints. Interestingly, the huge number of constraints means that just the task of finding a feasible starting point, i.e. a starting point that satisfies all the constraints, requires more than 48 hours for the old algorithm, after which the algorithm wrongly determines that there is no feasible starting point.

Finding a feasible point with the new algorithm requires roughly a minute, and we then take 500 iterations requiring about 20 minutes of computation time in the optimization algorithm.
TABLE II

RESULTS FROM THE OLD AND NEW ALGORITHM WHEN APPLIED TO THE REFLECTARRAY FROM SECTION III-C

\begin{tabular}{l|cc} 
& Optimization time & Optimum $F$ \\
\hline Old algorithm & $18: 33$ Hours & -0.76 \\
New algorithm & 9:52 Minutes & -0.76 \\
\hline
\end{tabular}

\section{Reflectarray}

Another topic of intense research is the direct [2] optimization of reflectarrays for contoured beam applications. In [3], a parabolic reflectarray with rectangular elements was optimized to fulfill the coverage requirements with a margin of $0.63 \mathrm{~dB}$, close to the performance of a shaped reflector which yields a $0.7 \mathrm{~dB}$ margin as reported in [4]. Interestingly, the optimization performed in [3] took more than 12 hours, after which the old algorithm appeared to have stalled at $0.63 \mathrm{~dB}$.

Revisiting the problem, we apply both algorithms to the problem. It turns out that the old algorithm was actually progressing beyond -0.63 , although very slowly, and attains the same minimum as the new algorithm at -0.76 , slightly better than the optimized shaped reflector in [4]. The results are detailed in Table II, demonstrating that the reduction in optimization time is a factor of 113 . The optimized reflectarray is shown in Fig. 2

\section{REFERENCES}

[1] J. Hald and K. Madsen, "Combined LP and Quasi-Newton Methods for Minimax Optimization," Mathematical Programming, vol. 20, pp. 49-62, 1981.

[2] M. Zhou, S. B. Sørensen, O. S. Kim, E. Jørgensen, P. Meincke, and O. Breinbjerg, "Direct Optimization of Printed Reflectarrays for Contoured Beam Satellite Antenna Applications," IEEE Transactions on Antennas and Propagation, vol. 61, no. 4, pp. 1995-2004, 2013.

[3] M. Zhou, S. B. Sørensen, and E. Jørgensen, "Parabolic Reflectarray for Broadband Telecom Satellite Applications with Low Cross-Polarization," in IEEE Antennas and Propagation Symposium, 2015.

[4] M. Zhou, O. Borries, and E. Jørgensen, "Design and Optimization of a Single-Layer Planar Transmit-Receive Contoured Beam Reflectarray With Enhanced Performance," IEEE Transactions on Antennas and Propagation, vol. 63, no. 4, pp. 1247-1254, 2015.
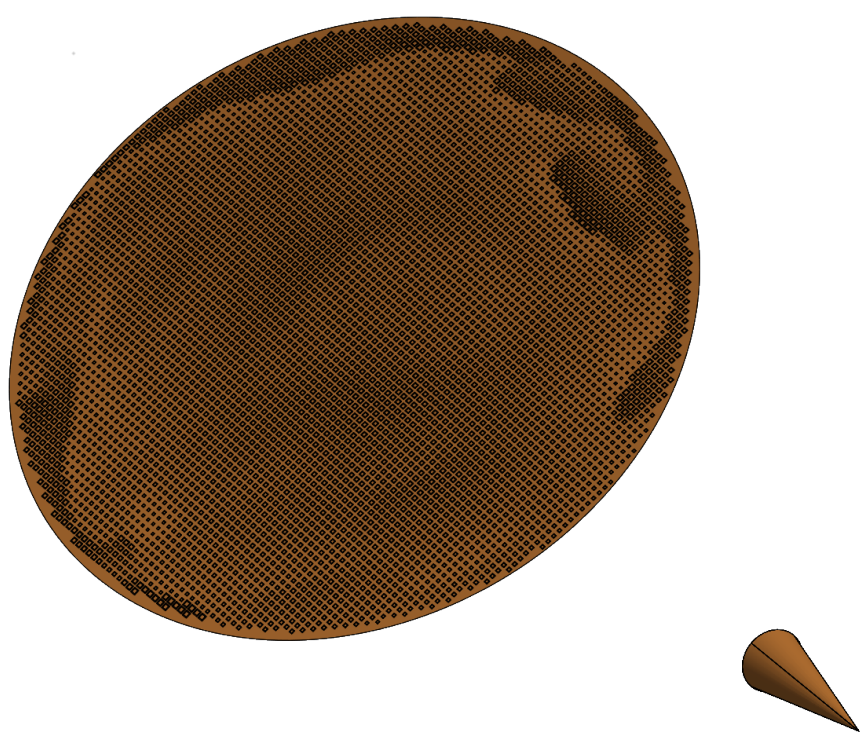

Fig. 2. The optimized reflectarray considered in Section III-C 